\title{
HEPARIN AS A RISK FACTOR FOR PERIGRAFT SEROMA COMPLICATING THE MODIFIED BLALOCK-TAUSSIG SHUNT
}

R. M. F. Berger, MD

G. Bol-Raap, $\mathrm{MD}^{\mathrm{b}}$

W. J. C. Hop, MSc, $\mathrm{PhD}^{\mathrm{c}}$

A. J. J. C. Bogers, MD, $\mathrm{PhD}^{\mathrm{b}}$

J. Hess, MD, $\mathrm{PhD}^{\mathrm{a}}$
Objective: The purpose of this study was to determine the risk factors associated with the occurrence of perigraft seromas complicating systemicto-pulmonary polytetrafluoroethylene grafts. Methods: Clinical and perioperative variables were reexamined, blinded for the outcome variable perigraft seroma, in 60 patients undergoing 67 consecutive graft procedures in a 3.5-year period. Results: Eight cases of perigraft seroma were diagnosed in six patients. Univariate analysis revealed age $(p=0.02)$, a diagnosis of pulmonary atresia with ventricular septal defect and systemic-pulmonary collaterals $(p=\mathbf{0 . 0 0 1})$, reimplantation of collaterals during the procedure $(p<0.001)$, and intravenous heparin administered after operation $(p<$ 0.0001 ) as risk factors for symptomatic perigraft seroma. Multivariable analysis defined heparin as the only significant factor associated with symptomatic perigraft seroma. Consolidation of the upper lobe on chest radiograph, ipsilateral to the shunt, directly after operation $(p=0.01)$, but especially 8 to 10 days after operation $(p<0.0001)$, or the need for prolonged drainage of pleural fluid $(p<0.0001)$ were correlated with the occurrence of perigraft seroma. Perigraft seroma led to four early rethoracotomies in three patients and to accelerated corrective surgery in three cases. Consolidation and absent perfusion of lung segments persisted in two patients. Conclusions: Our data suggest that the use of heparin leads to an increased risk of perigraft seroma, complicating systemic-pulmonary polytetrafluoroethylene grafts. Prolonged pleural drainage and/or postoperative consolidation of the upper lobe indicate the development of symptomatic perigraft seroma. Treatment is controversial and results are unpredictable. Expectative management seems to be justified so long as permitted by the clinical condition. (J Thorac Cardiovasc Surg 1998;116:286-93)
S stemic-pulmonary polytetrafluoroethylene (PTFE) grafts, like the modified Blalock-Taussig (MBT) shunts, are of major importance in the management of infants and children with various congenital heart diseases. ${ }^{1}$ The MBT shunt provides the possibility to

From the Departments of Pediatrics, Division of Pediatric Cardiology, ${ }^{\mathrm{a}}$ Cardiothoracic Surgery, ${ }^{\mathrm{b}}$ and Epidemiology and Biostatistics, ${ }^{c}$ Sophia Children's Hospital, University Hospital Rotterdam, Erasmus University Rotterdam, Rotterdam, The Netherlands.

Received for publication May 21, 1997; revisions requested August 4, 1997; revisions received March 4, 1998; accepted for publication April 13, 1998.

Address for reprints: R. M. F. Berger, MD, Division of Pediatric Cardiology, Sophia Children's Hospital, Dr Molewaterplein 60, 3015 GJ Rotterdam, The Netherlands; PO Box 2060, 3000 CB Rotterdam, The Netherlands.

Copyright (C) 1998 by Mosby, Inc.

$0022-5223 / 98 \$ 5.00+0 \quad \mathbf{1 2 / 1 / 9 0 8 6 9}$ create pressure- and volume-controlled pulmonary perfusion with preservation of distal flow in the subclavian artery. It has extended the surgical options in the treatment of children with cyanotic heart disease. Reported complications of the MBT shunt are thrombosis, infection, and pseudoaneurysm of the graft. ${ }^{2,3}$ An unusual and infrequently reported complication of the PTFE graft is a perigraft seroma." 4 This "clinicopathologic entity, manifested by a persistent, often enlarging, sterile fluid collection confined within a nonsecretory, fibrous pseudomembrane around a prosthetic graft" can seriously complicate the clinical outcome in patients. ${ }^{4}$ Because of compression of the lung, this complication may cause loss of lung tissue and functional pulmonary vasculature. Especially in patients with pulmonary atresia (PA), ventricular septal defect (VSD), systemic-pulmonary collateral arteries, and a hypoplastic pulmonary artery system, this is a major threat because the prerequisite for surgical 
Table I. Patient characteristics and diagnoses

\begin{tabular}{lcccc}
\hline \multicolumn{1}{c}{ Diagnosis } & $\begin{array}{c}\text { Patients } \\
(n)\end{array}$ & $\begin{array}{c}\text { PTFE } \\
\text { grafts }(n)\end{array}$ & $\begin{array}{c}\text { PGS }(\%) \\
(n)\end{array}$ & $\begin{array}{c}\text { Age (mo) } \\
(\text { range })\end{array}$ \\
\hline PA/VSD with & & & & \\
$\quad$ Reimplantation of SPCAs & 10 & 13 & $6(46)$ & $52(13-105)$ \\
$\quad$ Ligation of SPCAs & $8^{*}$ & 9 & $1(11)$ & $13(1-49)$ \\
Obstructed pulmonary flow in biventricular or univentricular heart & 22 & 23 & $1(4)$ & $1(0.1-9)$ \\
Tetralogy of Fallot & 12 & 13 & 0 & $0.7(0.1-4)$ \\
Hypoplastic pulmonary artery branch & 6 & 6 & 0 & $13(5-102)$ \\
Transposition of the great arteries and involuted LV & $\underline{3}$ & $\underline{3}$ & $\underline{0}$ & $\underline{1.5}(1-2)$ \\
Total & 60 & 67 & $9.1-105)$
\end{tabular}

$P A$, Pulmonary atresia; VSD, ventricular septal defect; $S P C A$, systemic-pulmonary collateral artery; $L V$, left ventricle; $P G S$, perigraft seroma; $P T F E$, polytetrafluoroethylene.

* One patient had two unifocalization procedures, with left-sided reimplantation of collateral arteries on one occasion and right-sided ligation of collateral arteries on the other occasion.

correction, being an adequately unifocalized pulmonary artery system, may be jeopardized. ${ }^{5,6}$ However, knowledge on perigraft seroma is limited; reports on management are infrequent, and early recognition may be difficult. In our series of 60 patients with 67 MBT shunts we diagnosed eight cases of symptomatic perigraft seroma in six patients. Clinical presentation, associated variables, and outcome will be described. Further, possible etiologic factors will be discussed.

\section{Patients and methods}

Data of all patients who underwent an MBT procedure with a PTFE graft in our institution between January 1993 and May 1996 were reexamined. Clinical variables (age, sex, diagnosis) and perioperative variables (diameter of the graft, reimplantation of collaterals into the native or newly reconstructed pulmonary artery in patients with PA and VSD, postoperative heparin administration) were retrospectively studied, with the occurrence of perigraft seroma as outcome variable. All chest radiographs, echographic studies, and computed tomographic (CT) and magnetic resonance imaging (MRI) scans were reexamined by investigators blinded for the outcome variable. All MBT shunts were constructed through a posterolateral thoracotomy at the fourth intercostal space. The MBT shunt was constructed either as an isolated procedure or as part of a unifocalization procedure. Interposition of a PTFE graft,* diameter $4 \mathrm{~mm}(n=2), 5 \mathrm{~mm}(n=61)$, or $6 \mathrm{~mm}(n=4)$, between the systemic and pulmonary circulations, was performed with a 7-0 polypropylene (Prolene; Ethicon, Inc., Somerville, N.J.) continuous suture for both anastomoses. The chest was drained by single-tube drainage. On postoperative day 1 , the decision was made whether to remove the drain. In the case of persistent pleural fluid leakage or pneumothorax, the chest tube was left in place as long as required. Anticoagulants were not administered routinely. Unifocalization procedures involving reimplantation of systemic-pulmonary collateral arteries on the native or newly constructed

* Gore-Tex graft, registered trademark of W. L. Gore \& Associates, Inc., Elkton, Md. pulmonary artery were regarded as an indication for anticoagulant therapy. In these cases, intravenous heparin administration was started directly after the operation, aiming at a two- to threefold increase in activated partial thromboplastin time. Subsequently, oral coumarin therapy was continued for 3 months. Also in cases of heparin treatment for thromboembolic events not related to the operative procedure, heparin therapy was continued in the postoperative period. In all patients, a chest radiograph was made within the first 24 hours after operation and 8 to 10 days after the procedure. In cases of persisting consolidation of the ipsilateral upper lobe or persisting leakage of pleural fluid ( $>48$ hours), thoracic echography was performed. A CT or MRI scan was performed only when clinical suspicion of a symptomatic perigraft seroma was present. The diagnosis of perigraft seroma was confirmed during subsequent surgical procedures.

Statistical analysis. The results were subjected to univariable and multivariable analyses. For continuous variables, comparisons between patients with and without perigraft seroma were made with the Student's $t$ test or the Mann-Whitney test when a nonparametric test was indicated. For categoric variables, comparisons were made with the Fisher exact test. Exact logistic regression analysis (LogXact; Cytel Software Corporation, Cambridge, Mass.) was used to evaluate the various factors simultaneously regarding the occurrence of perigraft seroma. It was verified that the findings were not essentially affected by the outcomes of the second graft procedure for those seven patients who had two graft procedures.

\section{Results}

In the study period, 67 MBT shunts with PTFE grafts were constructed in 60 patients. Of these, 36 were male. Seven of the 60 patients received two PTFE grafts: five because of consecutive unifocalization procedures on both sides, one because of pulmonary coarctation, and one because of thrombosis of the previous graft. Patient characteristics and diagnoses are shown in Table I. Eight of 60 patients died 3 to 273 days after the operation 
Table II. Univariate analysis of clinical and perioperative variables

\begin{tabular}{|c|c|c|c|c|}
\hline & $\begin{array}{c}P G S / \\
\text { total }\end{array}$ & OR & $95 \% C I$ & $\begin{array}{c}p \\
\text { Value }\end{array}$ \\
\hline \multicolumn{5}{|l|}{ Variable } \\
\hline \multicolumn{5}{|l|}{ Sex } \\
\hline Female & $1 / 26$ & 1 & & \\
\hline Male & $7 / 41$ & 5.0 & $0.6->100$ & 0.14 \\
\hline \multicolumn{5}{|l|}{ Age } \\
\hline$\leq 1$ year & $1 / 43$ & 1 & & \\
\hline$>1$ year & $7 / 24$ & 16.5 & $1.9->100$ & 0.002 \\
\hline \multicolumn{5}{|c|}{$\begin{array}{l}\text { Diagnosis of PA, VSD, } \\
\text { SPCAs }\end{array}$} \\
\hline No & $1 / 45$ & 1 & & \\
\hline Yes & $7 / 22$ & 19.5 & $2.2->100$ & 0.001 \\
\hline \multicolumn{5}{|c|}{ Graft diameter } \\
\hline $4 \mathrm{~mm}$ & $0 / 2$ & 0.0 & $0.0-11.8$ & 0.47 \\
\hline $5 \mathrm{~mm}$ & $6 / 61$ & 0.1 & $0.0-1.8$ & 0.07 \\
\hline $6 \mathrm{~mm}$ & $2 / 4$ & 1 & $1.5-18.2$ & \\
\hline \multicolumn{5}{|c|}{ Reimplantation of SPCAs } \\
\hline No & $2 / 54$ & 1 & & \\
\hline Yes & $6 / 13$ & 20.5 & $3.0->100$ & 0.0004 \\
\hline \multicolumn{5}{|l|}{ Heparin } \\
\hline No & $1 / 53$ & 1 & & \\
\hline Yes & $7 / 14$ & 46.8 & $5.0->100$ & $<0.0001$ \\
\hline \multicolumn{5}{|c|}{ Diagnostic variables } \\
\hline \multicolumn{5}{|c|}{$\begin{array}{l}\text { Consolidation on chest } \\
\text { radiography }\end{array}$} \\
\hline \multicolumn{5}{|c|}{$<24$ Hours postoperatively } \\
\hline No & $3 / 52$ & 1 & & \\
\hline Yes & $5 / 15$ & 7.8 & $1.3-58.7$ & 0.01 \\
\hline \multicolumn{5}{|c|}{ 8-10 Days postoperatively } \\
\hline No & $2 / 56^{*}$ & 1 & & \\
\hline Yes & $6 / 9$ & 50.0 & $5.6->100$ & $<0.0001$ \\
\hline \multicolumn{5}{|c|}{$\begin{array}{l}\text { Duration of chest-tube } \\
\text { drainage }\end{array}$} \\
\hline$\leq 48 \mathrm{hr}$ & $2 / 58$ & 1 & & \\
\hline$>48 \mathrm{hr}$ & $6 / 9$ & 47.7 & $5.8->100$ & $<0.0001$ \\
\hline
\end{tabular}

$O R$, Odds ratio; $95 \% C I$, 95\% confidence interval of odds ratio; $P A$, pulmonary atresia; $V S D$, ventricular septal defect; $S P C A$, systemic-pulmonary collateral artery.

*Two patients died before the eighth to tenth postoperative day.

(median 60 days). No patient died of MBT procedure-related causes. We diagnosed a total of eight symptomatic perigraft seromas in six patients. All seromas were confirmed during subsequent surgical procedures. The age was higher in patients diagnosed with perigraft seroma compared with those without perigraft seroma (median 28 months, range 0.2 to 67 months, versus median 2 months, range 0.1 to 105 months; $p=0.02$ ). Univariate analysis of the other variables is shown in Table II. Perioperative intravenous heparin therapy was administered in 14 procedures, either because of reimplantation of systemic-pulmonary collateral arteries $(n=8)$, arbitrarily estimated increased risk of thromboembolic
Table III. Multiple logistic regression analysis of various clinical and peroperative variables regarding the occurrence of PGS

\begin{tabular}{lccc}
\hline \multicolumn{1}{c}{ Variable } & $O R$ & $95 \%$ CI & $p$ Value \\
\hline Heparin & & & \\
$\quad$ No & 1 & & \\
$\quad$ Yes & 18.8 & $1.2->100$ & 0.02 \\
Diagnosis of PA, VSD, & & & \\
$\quad$ SPCAs & & & \\
$\quad$ No & 1 & & \\
$\quad$ Yes & 1.2 & $0.0->100$ & 1.0 \\
Graft diameter & & & \\
$\quad 4$ mm & 5.5 & $0.0->100$ & 1.0 \\
$\quad 5$ mm & 3.2 & $0.0->100$ & 1.0 \\
$\quad 6$ mm & 1 & & \\
Reimplantation of SPCAs & & & \\
$\quad$ No & 1 & & \\
$\quad$ Yes & 5.8 & $0.1->100$ & 0.78 \\
Sex & & & \\
$\quad$ Female & 1 & & \\
$\quad$ Male & 7.3 & $0.2->100$ & 0.47 \\
Age & & & \\
$\quad \leq 1$ year & 1 & & \\
$\quad>1$ year & 0.4 & $0.0->100$ & 1.0 \\
\hline
\end{tabular}

Reference categories are indicated with an odd's ratio of $1 . O R$, Odds ratio; $95 \% C I, 95 \%$ confidence interval of odds ratio; $P A$, pulmonary atresia; $V S D$, ventricular septal defect; $S P C A$, systemic-pulmonary collateral artery.

complications $(n=2)$, or perioperative thromboembolic events not related to the MBT shunt $(n=4)$. The heparin dose ranged from 6 to $20 \mathrm{IU} / \mathrm{kg}$ per hour; the duration of intravenous heparin administration ranged from 2 to 24 days (median 4.5 days). Four patients received coumarin without previous heparin therapy; symptomatic perigraft seroma developed in none of these patients. Table III shows the results of the multivariate analysis of various clinical and perioperative variables regarding the occurrence of symptomatic perigraft seroma. Adjusted for the other factors, heparin medication showed to be an independent predictor for symptomatic perigraft seroma (odds ratio 18.8; 95\% confidence interval 1.2 to $>100)$. None of the other factors showed a significant association with perigraft seroma. The same conclusions were reached when each of these variables was analyzed separately, only taking account of heparin use. All the significant (univariate) associations with perigraft seroma (Table II), months of age, the diagnosis of PA, VSD, and systemic-pulmonary collateral arteries, and reimplantation of systemic-pulmonary collateral arteries could be explained by the relation of these variables with heparin use. Although for chil- 
dren older than 1 year of age the percentage of heparin use was $42 \%$ (10/24 patients), this percentage was only $9 \%$ (4/43 patients) for infants who were younger than 1 year of age. Also, in case of reimplantation of systemic-pulmonary collateral arteries the percentage of heparin use was high, namely $62 \%$ (8/13 patients), whereas this percentage was $11 \%$ (6/54 patients) for other patients. In case of a diagnosis of PA, VSD, and systemic-pulmonary collateral arteries, heparin was used in $46 \%$ of the cases (10/22 patients), as compared with $9 \%$ (4/45 patients) for the patients with other diagnoses.

The duration of postoperative chest tube drainage for pleural fluid leakage was prolonged in patients with perigraft seroma compared with those without perigraft seroma (median 134 hours, range 20 to 600 hours, versus median 20 hours, range 0 to 120 hours; $p<0.0001)$. A chest radiograph revealing pleural fluid within 24 hours after the procedure was not correlated significantly with a diagnosis of perigraft seroma. However, three patients still showed pleural fluid on their chest radiograph 8 to 10 days after the procedure; perigraft seroma was diagnosed in two of these patients $(p=0.04)$. A consolidation of the ipsilateral upper lobe, directly after operation, but even more after 8 to 10 days, was associated significantly with the diagnosis of perigraft seroma (Table II). All patients who showed a persistent consolidation of the ipsilateral upper lobe or who required pleural fluid drainage by chest tube for more than 48 hours underwent echographic evaluation of the involved area $(n=12)$. In eight cases in which subsequently perigraft seroma was diagnosed, thoracic echography revealed an avascular, inhomogeneous echogenic area accompanied by compression of lung tissue and pleural fluid $(n=3)$; an avascular, echolucent cystic process $(n=2)$; an echolucent, pulsating mass $(n=1)$; isolated pleural fluid $(n=$ $1)$, or no abnormalities $(n=1)$. The relation of these abnormal areas to the graft could not be determined echographically. In the remaining four patients, thoracic echography revealed isolated compression of apical lung tissue, suggesting atelectasis. CT or MRI was performed in four patients in whom there was a clinical suspicion of perigraft seroma. In all four patients CT/MRI revealed a hypodens, cystic structure, adjacent to the graft in which no flow could be detected. In two of these four patients, such a structure was also detected adjacent to a previous PTFE graft. In retrospect, in both these patients the first graft procedure was followed by radiographically persistent consolida- tion of the ipsilateral upper lobe and one of the two had prolonged pleural fluid drainage. Echography in that period had revealed isolated pleural fluid in one case and no abnormalities in the other. So, six of the eight described cases of symptomatic perigraft seroma were documented by CT or MRI scanning.

Progressive respiratory distress forced us to reoperation in three patients, respectively 8,14 , and 25 days after the MBT procedure. In these patients, through a lateral rethoracotomy, the seroma was removed and the PTFE graft was left in place. In one of these patients the graft was additionally sealed with fibrin glue (Tissucol Duo 500; Immuno AG, Vienna, Austria). A consolidation of the upper lobe on chest radiograph recurred within 2 days in all three cases, leading to recurrence of respiratory distress in two patients. After the reintervention, all three patients required chest tube drainage for more than 48 hours because of pleural fluid production. In two patients the chest tube could be removed after 72 hours. Both patients gradually improved clinically. The third patient, in whom the graft was sealed with fibrin glue, needed a second rethoracotomy because of persisting pleural fluid production and respiratory distress, 35 days after the first rethoracotomy. A recurrent seroma was removed. This time the graft was sealed with microfibrillar collagen (Lyostypt; B. Braun Melsungen AG, Melsungen, Germany) and Histoacryl tissue adhesive (B. Braun Melsungen AG). At follow-up, of the patients who underwent one rethoracotomy, one patient showed spontaneous resolution of clinical and radiologic features and underwent corrective surgery 12 months after the MBT procedure. The other two patients both showed a persistent consolidation of the upper lobe on chest radiograph and an absent perfusion of the apical area on pulmonary artery angiography at 27 and 24 months of follow-up, respectively. In one of these two patients, surgical correction could be completed. In three other cases, the perigraft seroma led to accelerated surgical correction of PA, VSD, and systemic-pulmonary collateral arteries (two cases) and PA with intact ventricular septum (one case), with removal of the seromas and ligation of the grafts. These three patients recovered completely, with no sequelae at follow-ups of 4, 9, and 15 months, respectively. Finally, in two cases there was spontaneous resolution of clinical and chest radiographic features, except for a widening of the superior mediastinum on chest radiography as an isolated finding in one case. Elective correction was performed respectively 12 and 23 months after the MBT procedure. 


\section{Discussion}

The occurrence of a perigraft seroma can complicate the clinical outcome of patients undergoing an MBT procedure with a PTFE graft. The reported incidence of perigraft seroma needing treatment varies from 0.5 to $2.5 \% .^{2,3,7}$ However, the true incidence of perigraft seroma is unknown because a number of cases of perigraft seroma probably remain undetected. This is illustrated by a study in 29 adult, asymptomatic patients who underwent an abdominal CT scan after uncomplicated abdominal aortic reconstruction, in which seven cases of perigraft seroma were detected $(24 \%){ }^{8}$ Leakage of serous fluid from PTFE grafts is reported to have a significant higher incidence, up to $20 \% .^{7}$ In our series the incidence of a symptomatic perigraft seroma was $12 \%$ (95\% confidence interval $5 \%$ to $22 \%$ ). This high incidence may be related with the selection of our patient group. Because of a protocol adopted in 1991 in our institution for the performance of staged total surgical correction of patients with PA, VSD, hypoplastic pulmonary arteries, and systemic-pulmonary collateral arteries by subsequent unifocalization procedures, the patients comprise a relatively large and somewhat older group in our study.

Most frequently complicated by perigraft seroma are the knitted Dacron grafts, directly followed by PTFE grafts. ${ }^{4}$ Autografts do not seem to be affected by this complication. ${ }^{4,9}$ The cause of perigraft seroma is unknown. Various theories have been postulated, trying to explain the pathogenetic mechanism involved in the formation of perigraft seroma. The hydrophobic surface of PTFE is converted in a hydrophilic one, when in contact with blood or interstitial fluid, the so-called "wetting" process. 9 " 10 On the other hand deposition of proteins and attachment of fibrous tissue, both externally and internally, causes a "sealing" of the graft surface. ${ }^{4,9}$ In perigraft seroma the surrounding connective tissue fails to incorporate the graft, probably as a result of an imbalance between the "wetting" and "sealing" process. One reason for this imbalance may be mechanical forces, altering the architecture of the graft by increasing the distance between the PTFE fibers and thereby diminishing its water-repellent properties. The theoretic relationships among interfibrillar distance, intraluminal pressure, and graft leakage were demonstrated in a computer simulation by Tabata and coworkers. ${ }^{11}$ This would have direct implications for graft handling before and during the operation, such as stretching, bending, clamping, and compression of the graft. However, we found no differences in perioperative graft handling. According to this hypothesis, hemodynamic forces could be involved in the development of perigraft seroma. In our series age was associated with perigraft seroma in the univariate analysis. One could speculate that in older patients, with higher blood pressures, a higher mean pressure in the graft may lead to extravasation of fluid through the graft wall. However, age did not retain significance in the multivariable analysis, although in view of the wide confidence intervals its effect cannot be excluded from our data.

Histologic evidence of perigraft seroma has shown a fibrous pseudomembrane lining the seroma wall and immature fibroblasts lining the graft. In portions of the graft not involved with the seroma, a thicker and more densely adherent fibroblast layer has been observed. ${ }^{4}$ These observations have led to a theory of fibroblast inhibition as a mechanism leading to perigraft seroma formation. Studies of Sladen, ${ }^{12}$ Ahn, ${ }^{3,13}$ Schneiderman, ${ }^{14}$ and their associates suggest the presence of a humoral fibroblast inhibitor in the serum of patients in whom perigraft seroma is developing. This inhibitor could prevent the maturation and proliferation of perigraft fibroblasts, leading to poor graft incorporation. ${ }^{3,12}$ After removal of the grafts, the fibroblast inhibitory properties of serum of these patients decreased below detectable levels, suggesting that the graft may induce host production of such an inhibitor. ${ }^{3}$ Furthermore, it seems that this humoral factor can be cleared by plasmapheresis. ${ }^{12}$ A third cause for an disbalance between wetting and sealing may be the exposure of the graft to agents that diminish water repellency and thereby accelerate the wetting process. These agents include organic solvents, povidone iodium, antibiotic solutions, blood, and tissue fluids. ${ }^{9-11}$ In contrast to the findings of Leblanc and associates, ${ }^{7}$ our study suggests an important role for intravenously administered heparin in the development of perigraft seroma. Reimplantation of collaterals did not prove to be an independent risk factor. The same applied to the diagnosis PA, VSD, and systemic-pulmonary collateral arteries. One could speculate that heparin in the direct postoperative period impairs fibrin formation in the interstices of the PTFE graft wall and thereby disturbs the sealing process of the graft. Since the introduction, in the course of 1993, of our policy to provide heparin therapy for patients undergoing unifocalization procedures with reimplantation of systemic-pulmonary 
collateral arteries, we diagnosed eight cases of perigraft seroma in 60 patients in a 3.5-year period. Before this policy we did not observe any symptomatic perigraft seromas, despite numerous PTFE graft implantations. Although our policy induced a selection regarding the use of postoperative heparin, the multivariable analysis, taking account of the confounding factors, indicated that heparin use was an independent risk factor.

In our series, pleural fluid production, requiring chest tube drainage for more than 48 hours, was suggestive for the development of symptomatic perigraft seroma. A consolidation of the apical lung field at the side of the implanted graft on the chest radiograph, directly after operation, but even more 8 to 10 days after the procedure, was predictive of the presence of a perigraft seroma. However, differentiation with atelectasis, hematoma, or pseudoaneurysm can be difficult. ${ }^{7}$ Echocardiography has been reported to be useful in the identification of a thoracic mass and determination of its relation with arterial structures and the lung. ${ }^{15}$ In our experience, however, this differentiation appeared to be cumbersome. Thoracic CT/MRI scanning, performed in four of our patients, clearly demonstrated the presence of a cystic formation, without pulsatile flow, and its relation to the graft and the surrounding structures. The six perigraft seromas diagnosed with CT/MRI scanning were all confirmed surgically, indicating that $\mathrm{CT} / \mathrm{MRI}$ revealed no false-positive or false-negative findings in these patients. Reported complications of perigraft seroma include infection, graft thrombosis, and reoperation. ${ }^{3}$ Further, perigraft seroma can lead to compression and atelectasis of the upper lung lobe. Persisting compression of adjacent structures can result in a loss of functional lung tissue and the vasculature of the involved lobe, as shown by two of our patients. This complication is especially harmful in patients undergoing unifocalization procedures, aiming at an optimalization of the pulmonary vascular bed. ${ }^{5,6}$ Therapy of symptomatic perigraft seroma is controversial. Because of the high incidence $(65 \%)$ of spontaneous resolution of perigraft seroma, described in selected cases, ${ }^{4}$ expectative management seems to be justified. In symptomatic cases, however, intervention may be indicated because of progressive respiratory failure. Resection and aspiration of the perigraft seroma both are complicated by a high rate of recurrence and infection. ${ }^{4,16}$ Total graft removal, although effective, ${ }^{3,4}$ requires not only another surgical procedure to provide pulmonary flow but, even more important, use of new graft material. Other therapeutic options are based on enhancement or fortification of protein deposition to stop the leakage. ${ }^{9}$ This has been tried by wrapping the graft with absorbable collagen hemostat (Novacol Fibrillar) or collagen fleece soaked in fibrin glue, ${ }^{17,18}$ topical application of microfibrillar collagen or Histoacryl tissue adhesive, ${ }^{19,20}$ and intraluminal injection of a solution containing aprotinin, thrombin, and calcium chloride. ${ }^{21}$ As illustrated by our patients, these methods do not necessarily prevent recurrence or complications of the perigraft seroma. Two of our patients showed persisting compression of lung tissue and loss of functional pulmonary vasculature, despite surgical removal of the perigraft seroma. Finally, plasmapheresis has been reported as a strategy, based on the hypothesis of a humoral fibroblast inhibitor. ${ }^{12}$ Although this technique has shown to be successful in few patients, it has not been confirmed in clinical trials.

Limitations of the study. A retrospective study has inherent shortcomings. Heparin was not administered at random, but on arbitral grounds. More than one half of the patients received heparin therapy because of reimplantation of collaterals. However, the outcome of the multivariable analysis suggests patients who received heparin therapy had an increased risk of perigraft seroma. It has to be acknowledged that, because of a small number of events and, consequently, the wide confidence intervals, the effects of the other variables associated with perigraft seroma in the univariate analysis cannot be excluded. The study design does not allow a definite conclusion whether the relation between heparin use and perigraft seroma is causal or just coincidental.

In conclusion, our data strongly suggest that, administered postoperatively, intravenous heparin is associated with an increased risk for symptomatic perigraft seroma in patients undergoing MBT shunts with PTFE grafts. Although we recognize the potential beneficial effects of heparin in specific circumstances, we advocate to consider this possible adverse effect of heparin therapy after PTFE graft implantation, especially if associated with reimplantation of collaterals, when making decisions on its use. Consolidation of the upper lobe, ipsilateral to the shunt, on chest radiography directly after operation, but especially after 8 to 10 days, or prolonged drainage of pleural fluid may alert to the development of perigraft seroma. We believe CT or MRI scanning is the imaging technique of choice to 
diagnose suspected perigraft seroma. This complication of the MBT procedure may lead to loss of perfused lung segments. Treatment of perigraft seroma is controversial, and the results are unpredictable. Expectative management, even when accompanied by prolonged hospitalization, seems to be justified so long as it is permitted by the clinical condition.

\section{REFERENCES}

1. Gazzaniga AB, Lamberti JJ, Siewers RD, Sperling DR, Dietrick WR, Arcilla RA, et al. Arterial prothesis of microporous expanded polytetrafluoroethylene for construction of aortico-pulmunary shunts. J Thorac Cardiovasc Surg 1976; 72:357-63.

2. Borrero E, Doscher W. Chronic perigraft seromas in PTFE grafts. J Cardiovasc Surg (Torino) 1988;29:46-9.

3. Ahn SS, Machleder HI, Gupta R, Moore WS. Perigraft seroma: clinical, histologic, and serologic correlates. Am J Surg 1987;154:173-8.

4. Blumenberg RM, Gelfand ML, Dale WA. Perigraft seromas complicating arterial grafts. Surgery 1985;97:194-204.

5. Shanley CJ, Lupinetti FM, Shah NL, Beekman RH, Crowley DC, Bove EL. Primary unifocalization for the absence of intrapericardial pulmonary arteries in the neonate. $\mathrm{J}$ Thorac Cardiovasc Surg 1993;106:237-47.

6. Yagihara T, Yamamoto F, Nishigaki K, Matsuki O, Uemura $\mathrm{H}$, Isizaka $\mathrm{T}$, et al. Unifocalization for pulmonary atresia with ventricular septal defect and major aortopulmonary collateral arteries. J Thorac Cardiovasc Surg 1996;112:392-402.

7. LeBlanc J, Albus R, Williams WG, Moes CA, Wilson G, Freedom RM, et al. Serous fluid leakage: a complication following the modified Blalock-Taussig shunt. J Thorac Cardiovasc Surg 1984;88:259-62.

8. Qvarfordt PG, Reilly LM, Mark AS, et al. Computerized tomographic assessment of graft incorporation after aortic reconstruction. Am J Surg 1985;150:227-31.

9. McKay R. Shunts and security: the polytetrafluoroethylene leak. Cardiol Young 1995;5:6-8.

10. Bolton W, Cannon JA. Seroma formation associated with PTFE vascular grafts used as arteriovenous fistulae. Dial Transplant 1981;10:60-6.

11. Tabata R, Kobayashi T, Mori A, Matsuno S, Watarida S, Onoe $\mathrm{M}$, et al. A computer simulation of the plasma leakage through a vascular prosthesis made of expanded polytetrafluoroethylene. J Thorac Cardiovasc Surg 1993;105:598-604.

12. Sladen JG, Mandl MA, Grossman L, Denegri JF. Fibroblast inhibition: a new and treatable cause of prosthetic graft failure. Am J Surg 1985;149:587-90.

13. Ahn SS, Williams DE, Thye DA, Cheng KQ, Lee DA. The isolation of a fibroblast growth inhibitor associated with perigraft seroma. J Vasc Surg 1994;20:202-8.

14. Schneiderman J, Knoller S, Adar R, Savion N. Biochemical analysis of a human humoral fibroblast inhibitory factor associated with impaired vascular prosthetic graft incorporation. J Vasc Surg 1991;14:103-10.

15. Ozkutlu S, Ozbarlas N, Demircin M. Perigraft seroma diagnosed by echocardiography: a complication following Blalock-Taussig shunt. Int J Cardiol 1992;36:244-6.
16. Lewis $\mathrm{P}$, Wolfe JH. Lymphatic fistula and perigraft seroma [eitorial]. Br J Surg 1993;80:410-1.

17. Masiello P, Panza A, Di Benedetto G. Successful treatment of leakage of serous fluid following construction of a polytetrafluorethylene central shunt. Cardiol Young 1995;5:94-5.

18. Noyez L, Daenen W. The modified polytetrafluoroethylene Blalock-Taussig shunt: case report of an unusual complication. J Thorac Cardiovasc Surg 1987;94:634-5.

19. Rhodes VJ. Perigraft seroma: simple solution to a difficult problem [letter]. J Vasc Surg 1986;3:939.

20. Sim EK, Wong ML, Tan CT, Lee CN. Histoacryl tissue adhesive: an alternative means of stopping polytetrafluoroethylene graft sweating [letter]. J Thorac Cardiovasc Surg 1993; 106:1227-8.

21. Maitland A, Williams WG, Coles JG, Freedom RM, Trusler GA. A method of treating serous fluid leak from a polytetrafluoroethylene Blalock-Taussig shunt. J Thorac Cardiovasc Surg 1985;90:791-3.

\section{Commentary}

This is a clinical study of 67 consecutive modified Blalock-Taussig shunts in 60 patients. Eight cases of perigraft seroma developed in six of these patients, and this study is a retrospective evaluation of the variables that might be most associated with the development of this problem. The incidence of perigraft seroma in this study is rather high $(11.9 \%)$ compared with the incidence of $0.5 \%$ to $2.5 \%$ referenced in the manuscript. Furthermore, in our own practice, this is an extremely rare occurrence. In 117 aortopulmonary shunts placed since 1992 we have yet to recognize and document a single case of perigraft seroma. Therefore it is interesting that it is so "common" in the authors' experience. Furthermore, we frequently use heparin in the perioperative period for all patients who receive a polytetrafluoroethylene modified BlalockTaussig shunt; therefore it is concerning that the authors' retrospective review has led somehow to misleading conclusions. It is interesting to note that the patients in this study comprise a somewhat older group (mean age 9 months) who received generally larger shunts than what is seen more commonly in this country. For example, only two patients received $4 \mathrm{~mm}$ shunts and a vast majority of patients received shunts that were 5 or $6 \mathrm{~mm}$ in diameter. Interestingly, in the univariate analysis, shunt size did appear to be associated with perigraft seroma as did age, in that this finding was far more common in older patients (seven of eight cases in those more than 1 year of age). It is also interesting that the authors placed their shunts through thoracotomies. This might lead to the division of lymphatic vessels, which could also relate to the prevalence of perigraft seromas in their series. In our own personal experience, shunts have been placed through a median sternotomy since 1986, and the incidence of recognized perigraft seroma has been $0 \%$.

The authors have done the best they can with the data they have. They have a fairly large number of perigraft seromas and a relatively small number of patients, and it is interesting that this complication does appear more frequently in their patients who received heparin. Nevertheless, heparin is a widely recognized perioperative therapy for patients receiving aortopulmonary shunts. This is because the incidence of graft thrombosis is much higher in most 
experiences than the incidence of perigraft seroma. The authors administered heparin for a median of 4.5 days with a range between 2 and 24 days. This duration of heparin therapy may be longer than what is commonly practiced, and it may be that their association is with the duration of heparin therapy being long rather than with the use of heparin per se in the immediate postoperative period.

Although the authors point to an association with the use of heparin, there are several factors that may relate more specifically to the incidence of this complication. These may include the age of their patients, the size of the shunts being used, the duration of heparin therapy, the blood pressures that are generated in older patients, the placement of shunts through a thoracotomy, or even the off chance that some of the polytetrafluoroethylene used in this study may have been "resterilized." The association with heparin may be true from a multivariate standpoint but may not have really disclosed the actual mechanism by which this complication develops.
The authors are honest in discussing the limitations of this study and do make an important point regarding the presence of upper lobe consolidation persisting on the side ipsilateral to the shunt a week after the operation, which may be an important clinical sign that the presence of a perigraft seroma should be investigated. Perhaps the incidence of this complication is greater than we suspect, but the authors do point out that the patients in their series were all symptomatic and therefore raised suspicion by virtue of their clinical course. It is most important that the practicing pediatric cardiac surgeon evaluate the risks for and true incidence of perigraft seroma in his or her own practice in relation to the risks of not providing heparin for a limited time in the perioperative period after placement of an aortopulmonary shunt, especially shunts in the 3.5 and $4 \mathrm{~mm}$ diameters.

Ross M. Ungerleider, MD

Durham, N.C.

12/1/92127 